\title{
THE ANALYSIS OF UNDIAGNOSED MALIGNANCIES
}

\author{
Regina Kleina, Ivanda Franckevica, Maris Sperga, Daina Lutinska
}

Riga Stradins University, Department of Pathology, Riga Eastern Clinical University Hospital, Children Clinical University Hospital Riga, Latvia

\begin{abstract}
Among the pathology methods autopsy is also a valuable tool in evaluating diagnostic accuracy in oncology. Malignancies are undiagnosed due to the lack of some laboratory investigations, the absence of specific symptoms of cancers and the delayed treatment of them.

The aim is to analyze the reasons and the structure of undiagnosed malignancies in order to review the failure of their detection during the person's life and the hyper diagnosis of tumours in some hospitals of Riga (Latvia) in adults and children.
\end{abstract}

Key words: undiagnosed malignancies, diagnostic errors in children, adults

\section{MATERIAL AND METHODS}

We have analyzed 62 adult and 11 children cases with undiagnosed and misdiagnosed malignancies from the Pathology Center, the Riga Eastern Clinical University Hospital and the Pathology Bureau, the Children's Clinical University Hospital. The records of age, sex, location, the size of the tumor, the pathological diagnosis, complications and the evaluation of some clinical and laboratory data from the autopsy protocols and the clinical epicrisis were used. Morphological examinations were carried out with routine methods and with immunohistochemical reactions for the detection of $C K_{A E 1 / 3,} C D 20, C D 3$, WT1, vimentin and muscle antigens. The results were statistically evaluated using the Excel programmer.

Undiagnosed cancers in adults mostly were located in gastrointestinal system (62\%), haematopoetic organs (14\%) and lungs (6\%). In 
children during their life the diagnostics of the tumour was not carried out in hospital in the cases of soft tissue tumours (28\%), leukemia and lymphoma (27\%) and renal neoplasms (18\%).

Many of the protocols which included the clinical epicrisis showed that many symptoms suggesting malignancy were often missed and most commonly in our analyzed cases they were anemia, weight loss and pain symptoms.

The hyperdiagnosis of tumours was also proved in children and adults. The clinical presentation of the malignancy was misdiagnosed for a benign illness of the same organ.

The patient's delay in seeking medical help is also responsible for the late diagnosis of malignancies and therefore the screening of the population at risk must be introduced in Latvia on the primary level of health care for children and adults.

The most common reasons of discrepancy between the clinical and pathological diagnose was a short time of hospitalization (20\%), insufficient examination of the patient $(19 \%)$, objective diagnostic difficulties of tumours $(17 \%)$.

\section{INTRODUCTION}

Cancer has been one of the leading causes of death in the $21^{\text {st }}$ century. Cancers are misdiagnosed due to insensible results, poor laboratory investigations, delayed treatment and lack of medical care [4]. The failure to diagnose occurs in many cases due to the lack or mild symptoms in malignancies. Some neoplasm's symptoms often do not start until the disease has reached an advanced stage [5].

According to the recent studies in the United States and Europe around $12 \%$ of all the cases of cancer are misdiagnosed [9, 17]. The inability to identify a primary site of cancer poses many challenges. The primary site of cancer usually dictates the treatment, the expected outcome, and the overall prognosis.

The delayed treatment, the failure of diagnose at the beginning of neoplasm increases the mortality rate due to the oncology process $[15$, 20]. The early detection of cancer is vital for successful treatment, higher survival rates, and decreased medical costs. When cancer is diagnosed after the disease has progressed, more drastic forms of treatment are required. Intense cancer treatments (such as higher doses 
of radiation and chemotherapy) are not only painful and debilitating but cause added medical expenses $[1,13]$. The early diagnosis of cancer leads to a higher curative rate and a survival rate $[16,18]$.

The aim is to analyze the reasons and the structure of misdiagnosed cases of malignancies in order to review the failure of diagnosing malignant illnesses and the hyper diagnosis of malignancies in some hospitals of Riga in Latvia in adults and children.

\section{MATERIAL AND METHODS}

In order to review the rate and the occurrence of undiagnosed malignancies the research was carried out in the Pathology Center, the Riga Eastern Clinical University Hospital and in the Children's Clinical University Hospital.

From 2,481 autopsies made in the Pathology Center (Riga) in the years 2004-2010 in adults 62 cases with the discrepancy of clinical and post mortem diagnosis in oncology were found. But in the Children's Hospital from 1,705 post mortem examinations 11 cases were detected with undiagnosed tumours in the clinical departments. The recordings of age, sex, location, the size of the tumor, the pathological diagnosis, complications and the evaluation of some clinical and laboratory data from the clinical epicrisis were used. Morphological examinations were carried out with routine methods and with immunohistochemical reactions for the detection of $C K_{A E 1 / 3,} C D 20, C D 3, W T 1$, vimentin and muscle antigens. The results were statistically evaluated using the Excel programmer.

\section{RESULTS}

The analysis of 62 cases of adults and 12 children's cases of clinically undiagnosed or misdiagnosed oncology diseases was done. In adults from 62 cases in 53 persons tumours were revealed only at autopsy. The remaining 9 cases were hyper the diagnosis of cancer but the actual cause of death was another disease missed during clinical diagnosis. Of the undiagnosed cancers most commonly they were located in the gastrointestinal system, representing $62 \%$ of all the undiagnosed malignancies, in the haematopoetic system-12, $9 \%$, but others in some 
cases were in lungs, the urogenital system, soft tissues and the endocrine system (Figure 1).

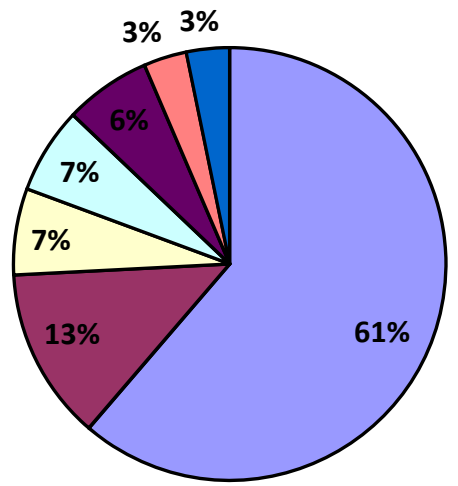

\begin{tabular}{|l|}
\hline$\square$ GIT \\
口Hematology \\
$\square$ Respiratory \\
$\square$ Urogenital \\
$\square$ Soft tissue \\
$\square$ Edocrine \\
$\square$ Reproductive \\
\hline
\end{tabular}

Figure 1. Undiagnosed malignancies of adults in the hospitals of Riga.

In 17 of the undiagnosed cancer cases, the diagnosis of the primary location of the cancer was incorrect with the mistaken clinical diagnosis that of malignancy from another location, which in reality were metastases, not the primary tumor. Distant metastases were recognized and diagnosed as the main clinical disease in 13 cases.

In autopsies we have already found dissemination of cancer without symptoms at the primary site but with clinical symptoms arising at the site of metastases.

Many of undiagnosed cases had a complication diagnosed as the main disease. Metastasis is one of the main complications of cancer in our analyzed group of patients. Symptoms, signs or another illness were diagnosed as the main disease in 25 cases. Clinical doctors, instead of malignancies, have put the diagnosis of: the coronary heart disease and atherosclerosis (9 cases), Jaundice ( 6 cases), acute abdomen ( 3 cases), bleeding ( 2 case), pneumonia ( 3 case) and anemia ( 2 cases). Many of the protocols which included the clinical epicrisis showed that many symptoms suggesting malignancy were often missed, e.g. severe anemia (14 cases), weight loss (8) and pain symptoms (5) were some of the signs and symptoms from the clinical epicrisis or the post mortem report which are associated with malignancies; 13 out of 30 cases of gastrointestinal system neoplasm and 5 out of 7 hematological malignancies 
had anemia fixed in the clinical epicrisis. Unexplained weight loss was mentioned in 8 of these misdiagnosed cases.

Of the 30 undiagnosed tumors which were located in the gastrointestinal system, 8 were detected in the stomach, 7 - in the pancreas, 6 - in the large intestines, 6 - in the liver and the biliary tract, 1 - in the small intestines and $2-$ in the esophagus.

Obvious presentations of malignancy were missed in some cases(e.g. in 4 cases of undiagnosed liver cancer, the patient presented with an enlarged liver weighing $2.7 \mathrm{~kg}$ during autopsy instead of $1.5 \mathrm{~kg}$ ); 5 cases of the missed diagnosis of pancreatic head tumors were diagnosed simply as "jaundice".

The number of undiagnosed cases of hematological neoplasms was 7 in total; 4 of the cases had obvious laboratory data suggesting an ongoing malignant process in the organism. (e.g. thrombocytopenia 66000 , hemoglobin 8.8 , erythrocytes 3.7 which supports the presence of anemia).

Enlarged lymph nodes and spleen (5 cases) should have suggested the physician at least to consider the diagnosis of hematological malignancy.

Histological variations of cancers were mainly different subtypes of adenocarcinomas and three cases were neuroendocrine tumors. The average mortality age for females was 68.4 but for males -61.1 years.

The situation with discrepancies between clinical and post mortem diagnosis in children is a little bit different. Mainly undiagnosed malignancies were found in the soft tissues (rhabdomyosarcoma, extraskeletal osteosarcoma) and kidneys (rhabdoid tumour, mezoblastic adenoma). Patients with renal tumours were treated from another kidney pathology in the hospitals of small towns; $27 \%$ of undiagnosed malignancies were leukemia, mainly acute ones and lymphomas at the average age of 7.6 years, Instead of hematological diseases clinical doctors diagnosed : meningococcal infection, nephroblastoma and the "enlarged kidney". 


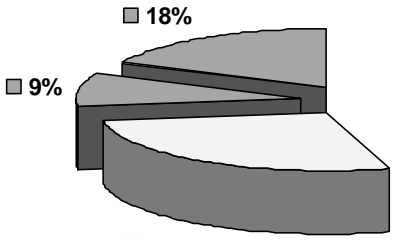

$\square \mathbf{2 7 \%}$

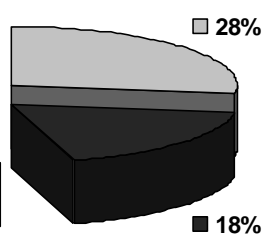

$18 \%$ $\square$ Soft tissue tumours

Renal tumours

$\square$ Leukemia, lyphoma

$\square$ Neuroblastoma

$\square$ Other malignancies

Figure 2. Undiagnosed malignancies in children (years 2004-2010 in Riga).

Another group of our analyzed cases constituted the hyper diagnosis or the misdiagnosis of malignancies.

In 14 adults and 1 child in clinical departments "malignancy" was diagnosed but the true pathological diagnosis was different nononcological pathology (Table 1). The true cause of death in these cases was a benign illness of the same organ, e.g., cholangiocarcinoma was diagnosed instead of the gallbladder stone disease. But in an 8-monthold girl brain tumour was misdiagnosed with the malformation of venous vessels and intracerebral bleeding. In all these cases there was too late hospitalization of the patient.

Table 1. Misdiagnosed malignancy cases in adults and children.

\begin{tabular}{|l|c|l|}
\hline Clinical diagnosis. & $\begin{array}{l}\text { Number } \\
\text { of cases }\end{array}$ & Post mortem diagnosis \\
\hline Gastric cancinoma & 4 & $\begin{array}{l}\text { Decompensated micronodular liver } \\
\text { cirrhosis }\end{array}$ \\
\hline $\begin{array}{l}\text { Malignant process of } \\
\text { mediastinum }\end{array}$ & 1 & $\begin{array}{l}\text { Decompensated micronodular liver } \\
\text { cirrhosis }\end{array}$ \\
\hline Cholangiocarcinoma & 2 & Gall stone disease \\
\hline Neoplastic syndrome & 2 & Atherosclerosis with intestinal gangrene. \\
\hline Gastric carcinoma & 1 & Anorexia nervosa \\
\hline Lung cancer & 3 & Lobar pneumonia. \\
\hline Thyroid cancer & 1 & Lobular pneumonia. \\
\hline $\begin{array}{l}\text { Brain tumor }(8- \\
\text { month-old girl })\end{array}$ & 1 & $\begin{array}{l}\text { Malformation of brain vessels with } \\
\text { cerebral bleeding }\end{array}$ \\
\hline
\end{tabular}




\section{DISCUSSION}

In Latvia, like in most of countries of the world, mortality from malignancies is on the second place after cardiac and vessel pathologies [14]. Regardless of the highly developed diagnostic techniques and medical health care systems, malignancies are commonly misdiagnosed and undiagnosed throughout the world and through centuries $[3,6,8]$. It was revealed that many symptoms such as pain, anemia and weight loss were among the leading symptoms that were misdiagnosed in these cases. Compared with other researches carried out in different regions of the world, these symptoms tend to play the leading role in most of the undiagnosed malignancy cases $[2,11,12]$. The bigest number of undiagnosed malignancies is from the gastro-intestinal system (62\%). This situation tends to appear similar when compared with the research carried in Brazil, Japan and Poland [2, 16, 21]. American researchers show that $2 \%$ of malignancies are of unknown primary origin. Misinterpretation of symptoms and signs contributes most to the undiagnosed malignancies $[1,18]$. This can be due to the lack of adequate diagnostic techniques [10] or the lack of medical care. But in our analyzed cases patients are looking for medical help at the last stages of tumours or even die at home without any medical aid. In the last years physicians pay more and more attention to nonspecific symptoms of malignancies and one of them is thrombosis and thrombembolism [7, 8, 19]. In our adult cases in one third part of persons had myocardial infarction-also as a manifestation of thrombosis in coronary arteries.

Very important criteria for autopsy discrepancies are described by Goldman L. et al. [4]:

1. Class I - the missed major diagnosis with the potential adverse impact on the survival and that would have changed management.

2. Class II - the missed major diagnosis with no potential impact on survival and that would have not changed therapy

3. Class III - the missed minor diagnosis related to the terminal disease but not related to the cause of death.

4. Class IV - the other missed minor diagnoses.

Our research proved that in $22.5 \%$ of adults' outcome of the disease could be changed by clinical doctors. But in paediatric cases practically all the patients were not curable at the time of hospitalization (class II). 
Decreased autopsy rates in countries, also in Latvia, should also be taken into consideration as not always the real reason of death can be detected by clinical practitioners.

\section{CONCLUSIONS}

1. Most commonly undiagnosed tumors were in the gastro-intestinal system at the stage $\mathrm{T}_{4} \mathrm{~N}_{2} \mathrm{M}_{1}$ and histologically they were adenocarcinomas in the patients with a short time of hospitalization.

2. Signs and symptoms such as weight loss, anemia and pain which were associated with malignancies were not taken into consideration by clinical doctors and the diagnosis of malignant tumour was missed and therefore everyone needs the improved screening and monitoring of oncology cases.

3. Despite high technologies of diagnostic equipment the discrepancy of clinical and pathological diagnoses are still present in the oncology cases and the most common reasons of it is a short time of hospitalization, the insufficient examination of the patient, the wrong formation of medical documentation and also the patient's delay in seeking medical help.

\section{REFERENCES}

1. Baron J.H. (2000) Clinical diagnosis and the functional of necropsy. J of Royal Society of Medicine, 93, 463-466.

2. Coradazzi A.L., Morganti A.L.C., Montenegro M.R.G. (2003) Discrepancies between Clinical diagnosis and autopsy findings. Brazilian J of Medical and Biological Research, 3, 36 (3): 385-391.

3. Damron T.A., Heiner J. (2000) Distant Soft Tissue Metastases: A Series of 30 New Patients and 91 Cases From the Literature. Ann Surg Oncol, 7, 7, 31-35.

4. Goldman L., Sayson R., Robbins S. et al. (1983) The value of the autopsy in three medical eras. N Engl J Med, 308-312.

5. Gordana J., Damir T., Jasminka J.R. (1999) Autopsy Findings and Clinical Diagnoses: Retrospective Study of 3,117 Autopsies, 40, 1, 623-629.

6. Grinberg L.T., Ferraz da Silva L.F., Galtarossa Xavier A.C. et al. (2008) Clinico-pathological discrepancies in the diagnoses of solid malignancies. Pathology - Research and Practice, 204, 12, 867-873. 
7. Karpman H.L. (2008) Venous Thromboembolism and Cancer. Internal Medicine Alert, 11, 29, 12-16.

8. Key N. (2008) Extensive screening superior to limited screening in detecting Trousseau Syndrome. Ann Internal Medicine, 149, 323-333.

8. Kirch W., Schaffi C. (1996) Misdiagnosis at a university hospital in four medical eras. Medicine, 75, 29-40.

9. Malvezzi M., A. Arfé A., Bertuccio P. Et al. (2011) European cancer mortality predictions for the year 2011. Ann Oncol, 22(4): 947-956.

10. Matasar M.J., Shi W., Silberstien J. et al. (2011) Expert second-opinion pathology review of lymphoma in the era of the World Health Organization classification. Annals of Oncology, 2, 23-29.

11. De Pangher Manzini V., Revignas M.G., Brollo A. (1970). Diagnosis of malignant tumors: comparison between clinical and autopsy diagnoses. Elsevier, New York, 100-121.

12. Pastores S.M., Dulu A., Voigt L. et al. (2007) Premortem clinical diagnoses and postmortem autopsy findings: discrepancies in critically ill cancer patients Critical Care, 11, 29-37.

13. Perkins G.D., McAuley D.F., Davies S., Gao F. (2003) Discrepancies between clinical and postmortem diagnoses in critically ill patients: an observational study. Critical Care, 7, 129-132.

14. Statistical overview on health and healthcare (2010) Health statistics and medical technology state agency of Latvia, 15-15.

15. Stevanovic G., Tucakovic G., Dotlic R. et all. (1986) Correlation of clinical diagnoses with autopsy findings: A retrospective study of 2,145 consecutive autopsies. Human Pathology, 17, 1225-1230.

16. Tachibana M., Takemoto Y., Monden N. et al. (1999) Clinicopathological features of early gastric cancer: Results of 100 Cases from a Rural General Hospital. Eur J Surg, 165, 319-325.

17. Tan W., MD, Shahab N., Amar S. (2008) Metastatic Cancer, Unknown Primary Site. http://emedicine.medscape.com

18. Tanne H. J. (1998) Cancer diagnosis is often missed. New York BMJ, 17, 317, 1033-1038.

19. White RH, et al (2005) Incidence of venous thromboembolism in the year before the diagnosis of cancer in 528,693 adults. Arch Intern Med, $165,1782-1787$.

20. Zarbo R.J., Baker P.B., Howanitz P.J. (1999) The autopsy as a performance measurement tool - diagnostic discrepancies and unresolved clinical questions. Archives of Pathology and Laboratory Medicine, 123, 191-198. 
21. Zubritsky A. (2007) Tumors of the digestive system by findings of post mortem examinations. Virchow's Archive, 451, 2, 213-217.

\section{Address for correspondence:}

Regina Kleina

Riga Stradins University

Department of Pathology, Riga Eastern Clinical University Hospital, Children Clinical University Hospital Riga, Latvia

2, Hippokrata Street, Riga, Latvia, LV 1038,

E-mail: rkleina@inbox.lv 